ANÁLISIS COMPARATIVO DE ESTRATEGIAS Y COMPETITIVIDAD ENTRE MIPYMES ASOCIADAS Y NO ASOCIADAS ${ }^{1}$

\title{
COMPARATIVE ANALYSIS OF STRATEGIES AND COMPETITIVENESS BETWEEN MIPYMES ASSOCIATES AND NO ASSOCIATES
}

\author{
Saulo Bravo García 2 , Jacinto Azcárate Serrano ${ }^{3}$, Alexander Rojas y Carlos Narváez Díaz \\ Corporación Universitaria Centro Superior - Unicuces. Cali-Colombia
}

RECIBIDO: Octubre 01 de 2014

ACEPTADO: Diciembre 09 de 2014

\section{RESUMEN}

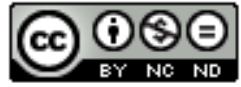

Este artículo es un estudio de carácter correlacional entre las empresas afiliadas y las no afiliadas a la Asociación Colombiana de las Micro, Pequeñas y Medianas Empresas (Acopi). Para lograrlo, se analizó la tendencia de ventas, las estrategias y la situación competitiva de las mipymes de la ciudad de Santiago de Cali. Se extrajo una muestra de 85 empresas aleatoriamente de una base de datos de la Cámara de Comercio de la ciudad de Cali y una muestra de 69 empresas de las registradas en Acopi; se aplicó un cuestionario cuyos datos se analizaron mediante el programa SPSS 22. En conclusión, las mipymes asociadas han tenido mayor favorabilidad en su desempeño de gestión estratégica y en las tendencias de crecimiento en ventas de un periodo a otro, a diferencia de las que no han contado con este modelo. Igualmente reciben un mayor número de beneficios relacionados con el fortalecimiento de sus recursos y capacidades debido a las sinergias que se realizan entre asociados y profesionales que conforman el gobierno interorganizacional en los modelos de asociatividad. Por lo mismo, es recomendable que las mipymes se vinculen a asociaciones que les puedan brindar apoyo estratégico para su competitividad.

Palabras clave: planeación estratégica, ventas, competitividad, cooperación, asociatividad.

\begin{abstract}
This article is a descriptive - comparative study between the affiliated companies and not affiliated to the Colombian Association of Small and Medium Enterprises (ACOPI). To achieve this, the strategies and the competitive situation of MSMEs of the city of Santiago de Cali was analyzed through the trend of sales. A sample of 85 (eighty five) randomly chosen companies from a database of the Chamber of Commerce of the city of Cali and a sample of 69 companies registered in ACOPI(Association of Small enterprises) ; a questionnaire whose data was analyzed using SPSS 22. In conclusion, the associated MSMEs have had a greater favorability in the performance of strategic management and growth trends in sales from one period to another, unlike those that haven't. They have relied on this model. In addition, they receive a greater number of related resources strengthening their capabilities due to the synergies that take place between partners and professionals who make up the inter-government partnership benefit models. Therefore, it is recommended that MSMEs be linked to associations that can provide them strategic support for competitiveness.
\end{abstract}

Keywords: Strategic Planning, Sales, Competitiveness, Cooperation, associativity.

Este Artículo se puede referenciar

Bravo, S., Azcárate, J., Rojas, A. \& Narváez, C. (2015) Análisis comparativo de estrategias y competitividad entre mipymes asociadas y no asociadas, en Desarrollo Gerencial Revista de la Facultad de Ciencias Económicas, administrativas y contables de la Universidad Simón Bolívar 7(1), 102-121.

\footnotetext{
${ }^{1}$ Proyecto de Investigación: análisis comparativo de estrategias y competitividad entre mypimes asociadas y no asociadas: una aproximaciòn en la ciudad de santiago de cali.

${ }^{2}$ Doctor en Economía y Administración, Director Unidad de Emprendimiento Universidad Santiago de Cali. sbravo@usc.edu.co, Investigacion@unicuces.edu.co.

${ }^{3}$ Doctor en Investigación Educativa, Director de Investigación Corporación Universitaria Centro Superior. Investigacion@unicuces.edu.co, Investigacion@unicuces.edu.co.

${ }^{4}$ en Mercadeo, docente y consultor Universidad Santiago de Cali. Investigacion@unicuces.edu.co

${ }^{5}$ Maestría en Educación, Director de Planeación Corporación Universitaria Centro Superior. planeacion@unicuces.edu.co, Investigación@unicuces.edu.co.
} 


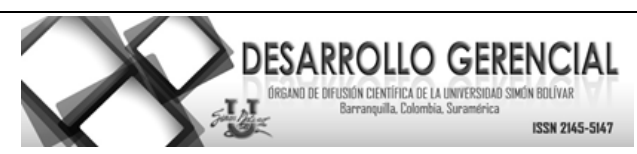

\section{1.- INTRODUCCIÓN}

La micro, así como la pequeña y la mediana empresa (Mipymes) han sido el centro de atención de gobiernos en los últimos años, por su condición de generadoras de riqueza colectiva, además de la oportunidad que al mercado brindan con su presencia y aportes al crecimiento socioeconómico de una región. En este sentido, se han convertido en punto de referencia para investigadores a nivel global, con el propósito de encontrar fórmulas que refuercen sus capacidades innovadoras.

En la presente investigación se esboza un análisis descriptivo-comparativo entre Mipymes asociadas y no asociadas en la ciudad de Santiago de Cali, buscando con ello identificar la importancia o no de hacerlo como herramienta estratégica para la competitividad; teniendo en cuenta que este tipo de empresas representan el 80,8\% del empleo en Colombia, según el Ministerio de Comercio, Industria y Turismo (s.f.), por lo que se hace necesario identificar la relevancia o no de la asociatividad de dichas empresas.

Una mirada general, a manera de perfilar las mipymes como objeto de investigación, puede ayudar a comprender la relevancia de reconocer el componente de asociatividad como estrategia para la competitividad. Los siguientes componentes dan una idea clara al respecto:

$\checkmark$ El tejido empresarial en Colombia refleja un dominio de representatividad de las mipymes que generando 37\% del PIB nacional equivalen al 99,7\% de las unidades empresariales registradas en Colombia; 650 mil mipymes están inscritas en el sistema de seguridad social. Además, como ya se mencionó, el Ministerio de Comercio, Industria y Turismo (s.f.) señala que proveen el 80,8\% del empleo en Colombia. Son definidas como unidades económicas de 10 (micro), no más de 50 (pequeña) y hasta 200 (la mediana) trabajadores.

$\checkmark$ En América Latina las mipymes resultan importantes desde el punto de vista de la generación de empleo y de ingresos para la población y representan el $90 \%$ del total de empresas constituidas (Saavedra y Hernández, 2008). No obstante, la existencia de debilidades de carácter estructural que padecen sigue afectando su supervivencia y mermando su competitividad.

La primera parte de este artículo se centra en una aproximación teórica al concepto de planeación estratégica, cooperación y competitividad, mediante la revisión de la literatura; en la segunda se reflexiona acerca de la metodología y el tipo de estudio y diseño de la muestra utilizada; la tercera presenta el análisis e interpretación de los resultados y en la cuarta parte se obtienen las conclusiones e implicaciones para la gestión del tema analizado. Es por ello que esta investigación pretende establecer la relevancia o no de la condición de estar o no asociadas, como estrategia para la competitividad. 


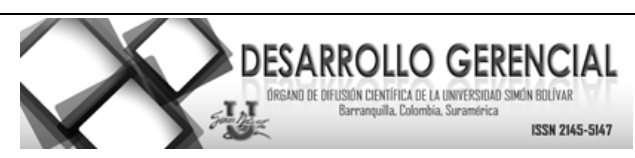

\section{Fundamentos teóricos}

La fundamentación sobre la competitividad en las mipymes es un tema que se aborda desde el desarrollo empresarial y cuyo abordaje se hace desde la literatura científica. Dicho concepto guarda estrecha relación con los procesos de gestión estratégica en función de los logros de crecimiento, través de los análisis de los factores externos e internos. En este sentido, Grant (citado en Gálvez, E., Cuéllar, K., Restrepo, C., Bernal, C. y Cortés, J., 2012), señala: "Desde esta perspectiva, la clave del éxito competitivo se sitúa en elementos pertenecientes a la propia empresa, una idea que conceptualmente ha propiciado el desarrollo de la Teoría de Recursos y Capacidades" (p. 17)

\section{Entorno competitivo}

Un hecho relevante del entorno competitivo es considerar que los mercados no son organismos amorfos, sino que presentan estructuras las cuales, a su vez, inciden en la conducta de los mercados y, por consiguiente, en sus resultados. Dicha propuesta está sustentada por el paradigma propuesto por Mason y Bain (citados en González J. y Rosero, 2011). Al respecto, Gálvez et al. (2012) señalan lo siguiente:

La base de la economía industrial se asienta en el conocido paradigma estructuraconducta-resultados según el cual el tipo de estructura de los mercados determina el comportamiento de las empresas que los componen, lo que en último caso determina sus resultados. Por lo [(...) tanto,] es relevante considerar que el carácter circular de este paradigma puede suponer que el comportamiento altere la estructura y los resultados organizacionales.

En este orden de ideas, las empresas se tornan competitivas toda vez que hagan frente a lo que Porter (1980) denominó: las cinco fuerzas competitivas. Este modelo se basa en: "1) la amenaza de productos sustitutivos; 2) la amenaza de los competidores potenciales; 3) el poder de negociación de los proveedores; 4) el poder de negociación de los clientes y 5) la rivalidad entre los competidores" (Gálvez et al., 2012, p. 20).

Este modelo parte de dos premisas fundamentales: la estructura de la industria y el perfil competitivo; en el caso de la primera, determina la naturaleza de la competencia, siendo este el principal determinante de los beneficios de las empresas (Hill \& Deeds, 1996).

Desde la segunda premisa la empresa asumirá el perfil competitivo que mejor se adapte a las características estructurales de la industria, es decir, establecerá su estrategia de negocio sobre la base de la situación estructural de sus mercados. Sin embargo, se requiere de la aplicación de un tipo de plan que 


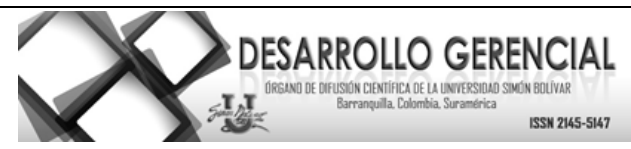

contribuya a un proceso integrador y brinde alternativas efectivas. En este sentido, para el análisis se ha considerado hacer un acercamiento desde la perspectiva de la planeación estratégica y estrategia.

\section{Planeación estratégica y estrategia}

"La planeación estratégica tiene por objeto fijar un curso concreto de acción, estableciendo los principios que habrán de orientarlo, la secuencia de operaciones, la determinación del tiempo y los recursos necesarios para su realización" (Gálvez et al., 2012). Para las empresas, fijar un curso de acción que sea concreto se hace indispensable puesto que ello determinará su competitividad y su permanencia en el mercado.

Al respecto, Armijo (2009) señala con relación al establecimiento de objetivos estratégicos que ello hace referencia a las grandes decisiones, permitiéndose con ello materializar la misión y la visión de la empresa.

En este sentido, el proceso de planeación estratégica implica la realización de una serie de actividades formalizadas por parte de las empresas, encaminadas a identificar objetivos, metas, analizar el contexto externo y los recursos internos, para así conseguir identificar oportunidades, amenazas del entorno y determinar fortalezas, debilidades de la organización. Este proceso permitirá a la empresa formular estrategias adecuadas a sus objetivos, teniendo en cuenta la situación interna y externa (Gálvez et al. 2012, p. 21).

Por otro lado, la estrategia es la fórmula que le define a la empresa en qué va a competir, es decir, cómo va a decidir las políticas necesarias para alcanzar los objetivos; en este sentido, para la ejecución de una estrategia se debe tener en cuenta su planeación con sus respectivos análisis, que como lo señala Armijo (2009): "la Planificación Estratégica consiste en un ejercicio de formulación y establecimiento de objetivos de carácter prioritario, cuya característica principal es el establecimiento de los cursos de acción (estrategias) para alcanzar dichos objetivos" (p. 5).

La estrategia, entonces, va a conducir al posicionamiento de la empresa en el entorno competitivo del cual hace parte estructural, ofreciéndole ventajas sobre sus competidores. Grant (1996) manifiesta que sin una competencia, la estrategia crecería de interés. No habría necesidad de establecer objetivos en función de prever el entorno y la planificación del empleo de los recursos.

Formular la estrategia competitiva implica hacer un análisis interno que evidencie tanto los puntos fuertes como los débiles, los cuales conforman el perfil de activos y habilidades que refleja cuál es su posición con relación a sus competidores. De igual forma, como lo sugiere Porter (1980), también se hace necesario el análisis externo tanto del sector industrial como del entorno circunscrito a la empresa. 


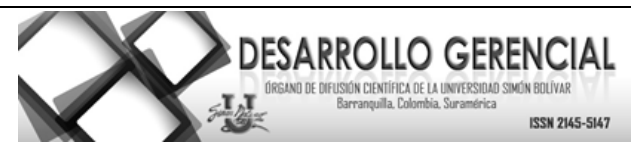

Para las empresas, es evidente la necesidad e interés en conocer aquellas estrategias que permitan alcanzar mejores resultados a la par de la mejora en su competitividad. Sin embargo, autores como Miles y Snow (citados en Pleshko, 2006 y Wright et al. 1991) manifiestan que no hay una relación directa entre la estrategia seguida por la empresa y el éxito competitivo alcanzado. Por su parte, Spanos et al. (2004); Aragón y Sánchez (2005) y Rubio y Aragón (2009) encuentran que los rendimientos que obtienen las empresas dependen de la orientación estratégica que se ha de seguir.

Una estrategia empleada con mayor frecuencia es la propuesta presentada por Miles y Snow (citados en Pleshko, 2006). Esta clasificación es una de las más utilizadas y aceptadas en la literatura por su visión de la organización McDaniel y Kolari (citados en Gnjidić, 2014) y por centrarse en el proceso de interacción dinámica de la empresa con el entorno, lo que hace que se tenga en consideración la relación entre recursos externos e internos O’Regan y Ghobadian (citados en Gálvez et al., 2012).

Miles y Snow (citados en Gálvez et al., 2012) se basan en 3 premisas: la primera señala que las empresas con mayor éxito son las que desarrollan un enfoque sistemático e identificable en cuanto a la adaptación en su entorno; una segunda premisa hace referencia al hecho que se puede identificar cuatro tipos de orientaciones estratégicas en cada sector; las empresas se clasifican en: exploradoras, analizadoras, defensivas y reactivas (Pleshko, 2006) y una tercera premisa hace referencia a que cualquiera de las tres orientaciones -exploradora, analizadora o defensiva- puede conducir a un buen resultado empresarial, dado que la cuarta orientación - estrategia reactiva- es asumida con falta de consistencia y, por lo tanto, tendrá menores rendimientos que las otras tres (Gálvez et al. 2012).

Este tipo de organizaciones son incapaces de responder a los exigentes cambios de su entorno lo que las hace inestables e inconsistentes, pues según lo argumentan teóricos como Slater y Narver (1993) y empíricos como Wright et al. (1991) carecen de estrategias consistentes y claras.

Con relación a las tres restantes orientaciones estratégicas: defensiva, exploradora y analizadora, Gálvez et al. (2012) manifiestan lo siguiente:

La orientación estratégica defensiva corresponde a empresas que se concentran en un estrecho y limitado ámbito del producto-mercado; expertas en un área determinada, no buscan nuevas oportunidades fuera de su negocio, sino que tratan de proteger su cuota de mercado. Necesitan pocos ajustes en tecnología, estructura o procesos. Están orientadas internamente y enfatizan la reducción de costes y el incremento de la eficiencia. El patrón estratégico de los exploradores es el opuesto al de los defensivos, buscan continuamente nuevas oportunidades de mercado mediante procesos de innovación y desarrollo de productos. Actúan rápidamente ante las tendencias emergentes del entorno y suelen propiciar cambios en el sector (p. 21). 


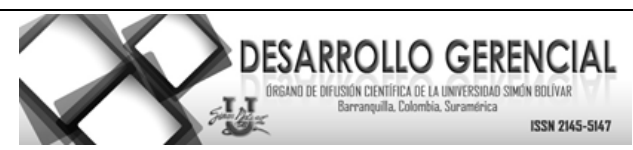

Los mismos autores manifiestan que dentro de las estrategias de tipo defensivas disponen de tecnologías muy flexibles, por lo que se puede afectar su eficiencia interna.

Por otro lado, las organizaciones analizadoras son una resultante de las estrategias defensivas y exploradoras, en tanto que actúan de modo defensivo o explorador "dependiendo del entorno en el que se encuentren y del equilibrio eficiencia-innovación que requieran" (Gálvez et al 2012).

En condiciones normales de los entornos, las organizaciones operan de manera rutinaria, formal y eficiente a través de estructuras y procesos formalizados; en entornos turbulentos son seguidoras de los competidores más innovadores que tratan de adoptar con rapidez las ideas que parezcan más prometedoras.

Por otro lado, es importante señalar que existe la necesidad de buscar aliados y establecer sinergias entre las organizaciones como estrategias en los procesos de desarrollo, de tal forma que permita el crecimiento de las empresas tanto a nivel local como regional, esto es, trabajando de manera cooperativa.

\section{Cooperación}

La construcción de alianzas a manera de cooperación entre empresas, supone un procedimiento a través de fórmulas jurídicas o bien con acuerdos explícitos o tácitos en donde las partes ofrecen sus capacidades y recursos, pero que sin llegar a fusionarse establecen un cierto grado de interrelación que permita incrementar las ventajas competitivas.

Como alternativa estratégica, es necesario comprender que la cooperación permite mantener independencia jurídica y patrimonial, a la vez que ofrece la posibilidad de alcanzar el tamaño competitivo en ciertas actividades. En este sentido, la cooperación no es otra cosa que unir fuerzas donde cada empresa dentro de ciertas alianzas, ofrece su capacidad competitiva.

En este sentido, Montes y Sabater (citados en Gálvez et al. 2012) manifiestan que:

... Pese a la importancia teórica y práctica de la cooperación, y a pesar del apoyo institucional a la misma entre [mipymes] (financiando los gastos del proceso en muchos casos), [estas] todavía [(...) miran con recelo el uso de tal] arma estratégica, debido a la reticencia a compartir sus decisiones con otras empresas, al excesivo individualismo de sus propietarios, a la desconfianza en el resto de cooperantes o por sentirse incapacitadas para desarrollar el acuerdo (p. 22).

Sin embargo, autores como Martín y Gaspar (2005) y Montoro (2005), (citados en Ojeda, 2009), consideran que la cooperación entre las instituciones se pueden convertir en un medio para desarrollar sinergias. 


\section{2.- MÉTODO}

\section{Diseño}

El estudio desarrollado fue de tipo correlacional, en tanto que como lo manifiestan Hernández et al. (2014): pretende "conocer cuál es la relación o grado de asociación que exista entre dos o más conceptos, categorías o variables en una muestra o contexto en particular" (p. 93); se compara los procesos formales estratégicos y la competitividad entre las empresas afiliadas y las no afiliadas a la Asociación Colombiana de las Micro, Pequeñas y Medianas Empresas (Acopi);

\section{Participantes}

La población pertenece a las mipymes no asociadas en Santiago de Cali y asociadas en el gremio de Acopi de la misma ciudad, de allí fueron tomadas dos muestras de manera aleatoria tanto a partir de una base de datos de la Cámara de Comercio con 1700 empresas no asociadas como de las registradas en Acopi. Se definió entonces una muestra de 85 empresas no asociadas, y del sector Acopi, 69; se aplicó un cuestionario, cuyos datos se analizaron mediante el programa SPSS 22.

\section{Instrumento}

Para la recolección de la información se adaptó el cuestionario desarrollado por la Red Faedpyme. La codirección internacional de la Red trabajó en conjunto con la Universidad del Valle en la adaptación del cuestionario a la realidad colombiana. Para su adaptación se realizaron pruebas que permitieran darle validez y confiabilidad (Gálvez et al 2012).

\section{Procedimiento}

Para la recolección de la información se procedió a contactar las Mipymes no asociadas mediante el principio del muestreo sistemático que consiste en que los elementos de la población están ordenados en una base de datos. Luego se procedió a visitar 200 empresas de las cuales se obtuvo respuesta de 85 . Por otro lado se realizó visita al gremio Acopi donde se tuvo en cuenta la población de Mipymes asociadas, 300 según información suministrada. De esta población, se obtuvo respuesta de 69 Mipymes. Una vez obtenida respuesta de las Mipymes, se concertó las correspondientes citas para aplicar el cuestionario, de manera individual a cada uno de los propietarios o administradores. Posterior a la recolección de la información, se registró en la base de datos del programa SPSS 17, con el cual se realizó el análisis correspondiente. 


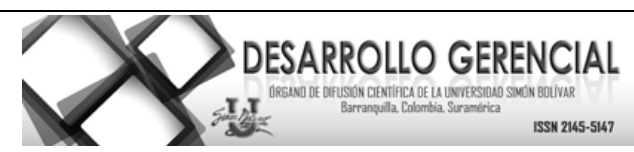

\section{3.- RESULTADOS}

Tabla 1. Caracterización de los sectores al que pertenecen las Mipymes analizadas Sector empresarial

\begin{tabular}{|l|c|c|}
\hline \multicolumn{1}{|c|}{ SECTOR } & Frecuencia. & Porcentaje \\
\hline Productos alimenticios y bebidas & 29 & $34 \%$ \\
\hline Industria textil de la confección, peletería y cuero & 2 & $2 \%$ \\
\hline Industria de madera y corcho & 1 & $1 \%$ \\
\hline Industria de papel, edición o artes gráficas & 1 & $1 \%$ \\
\hline Industria química & 3 & $4 \%$ \\
\hline Fabricación de productos de caucho y materiales plásticas & 0 & $0 \%$ \\
\hline Fabricación de otros productos minerales no metálicos & 0 & $0 \%$ \\
\hline Metalurgia, fabricación de productos metálicos & 3 & $4 \%$ \\
\hline Industria de la construcción de maquinaria & 0 & $0 \%$ \\
\hline Material y equipo eléctrico, electrónico y óptico & 0 & $0 \%$ \\
\hline Fabricación de vehículos de motor & 0 & $0 \%$ \\
\hline Fabricación de muebles & 1 & $1 \%$ \\
\hline Construcción & 0 & $0 \%$ \\
\hline Comercio al por mayor & 5 & $6 \%$ \\
\hline Comercio al por menor & 21 & $25 \%$ \\
\hline Hostelería y restaurantes & 3 & $4 \%$ \\
\hline Transporte terrestre, marítimo y aéreo & 3 & $4 \%$ \\
\hline Actividades informáticas & 8 & $9 \%$ \\
\hline Servicio a empresas, profesionales, científicos y técnicos & 5 & $6 \%$ \\
\hline & $\mathbf{8 5}$ & $\mathbf{1 0 0 , 0} \%$ \\
\hline
\end{tabular}

Fuente: los autores (2015)

\section{Gráfica 1. Sector donde se ubica la empresa}

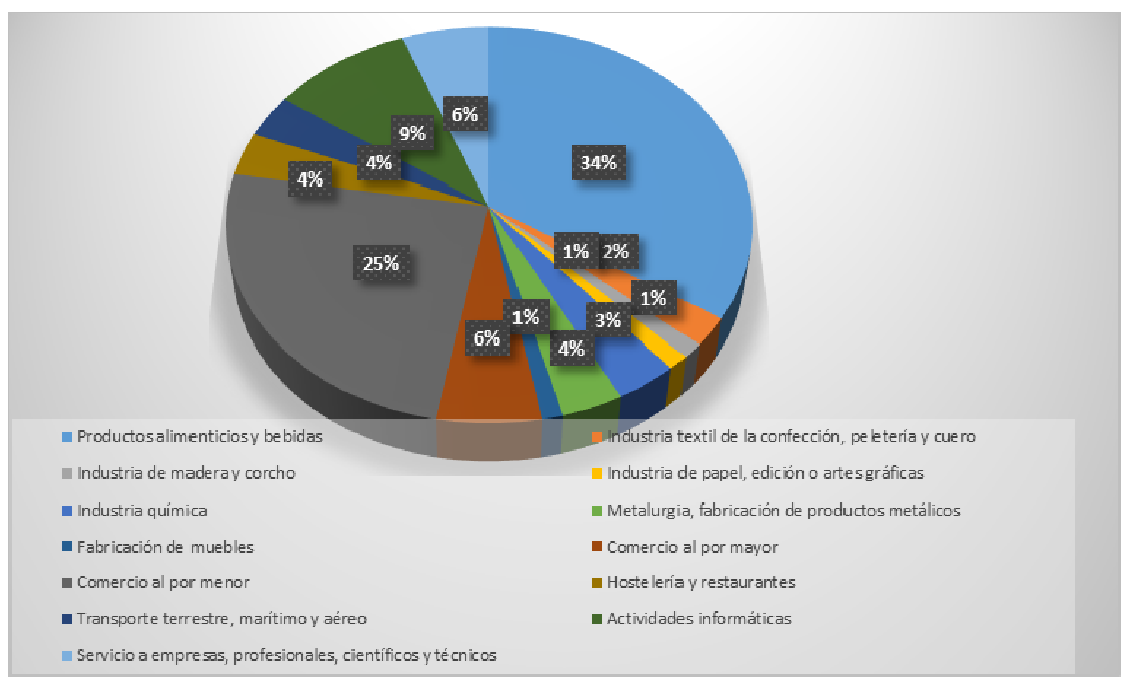

Fuente: los autores. 


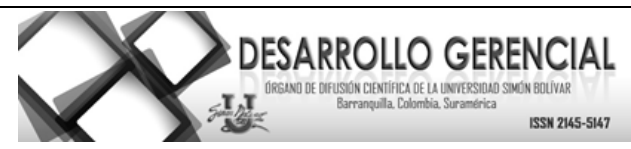

Tanto en la gráfica 1 como en la tabla 1 se aprecia que pertenece al sector de los productos alimenticios y bebidas el 34\% de las 85 empresas analizadas en las mipymes no asociadas; el 34\% pertenecen al sector de los productos alimenticios y bebidas; el 25\%, al del comercio minorista; un 6\%, del comercio mayorista; un 9\%, al sector de actividades informáticas; un 6\% corresponde a prestación de servicios a empresas, profesionales, científicos y técnicos y un $20 \%$ reparte entre el resto de sectores de los 19 analizados de menor representatividad de acuerdo a los resultados.

Mientras tanto en las 69 empresas asociadas a Acopi, se refleja una distribución diferente de los sectores reportados entre las no asociadas así: un 8.70\% corresponde a los productos alimenticios y bebidas; un 4,35\%, al comercio al por mayor; un $1.35 \%$, a actividades de informática y un $18.84 \%$ son servicios a empresas, profesionales científicos y técnicos, el más representativo, seguido de otros como fabricación de productos de caucho y materias plásticas en un 13.04\%; metalurgia, fabricación de productos metálicos, un $11.59 \%$; industria textil de la confección, papelería y cuero, $8.70 \%$; industria de papel, edición o artes gráficas, un 5.8\%; un 27.27\% se reparte entre el resto de sectores de los 19 analizados de menor representatividad de acuerdo a los resultados.

Con lo anterior, en el caso de las empresas no asociadas se podría afirmar que una tendencia de surgimiento de mipymes que nacen por iniciativa de emprendedores lo hacen por necesidad entendida como aquella situación en la cual el individuo se ve obligado a iniciar su propia actividad productiva porque no encuentra una mejor opción de trabajo; al respecto, en un estudio de (GEM) Global Entrepreneurship Monitor (2013) muestra que un $18.4 \%$ lo hace por necesidad y en ocasiones sin procesos formales.

Por otro lado, en las gráficas siguientes se puede verificar que las mipymes organizadas en un modelo de asociación presentan un comportamiento diferente con tendencia a un crecimiento más estable y que se pueden relacionar con emprendedores adoptando un criterio de oportunidad parcial o entendida como el aprovechamiento de una coyuntura de empresa, en la que el 51.3\% tiende a involucrarse según revela el mismo estudio (Varela y Liyis, 2013).

En este sentido, se realizará un análisis de las empresas que siguen un modelo de planeación estratégica y las que no la usan estando o no en un sistema asociativo, donde se pretende evaluar cuáles reflejan un mayor crecimiento e innovación y el consiguiente crecimiento económico. En consecuencia, se analizarán las preguntas relacionadas en el estudio con proceso de planeación estratégica. 
Tabla 2. Procesos formales estratégicos (no asociadas)

\begin{tabular}{|l|c|c|}
\hline & Frecuencia & Porcentaje \\
\hline Sí & 37 & $44 \%$ \\
\hline No & 48 & $56 \%$ \\
\hline TOTAL & 85 & $100,0 \%$ \\
\hline
\end{tabular}

Fuente: los autores.

En la tabla 2, se evidencia que en el $44 \%$ de las empresas no asociadas se realizan procesos de planeación estratégica en el desarrollo de sus actividades y con tendencias a corto plazo mientras que el 56\% no realizan ningún tipo de planeación. En la gráfica 2 se refleja la siguiente situación.

Gráfica 2. Planteamiento estratégico formal

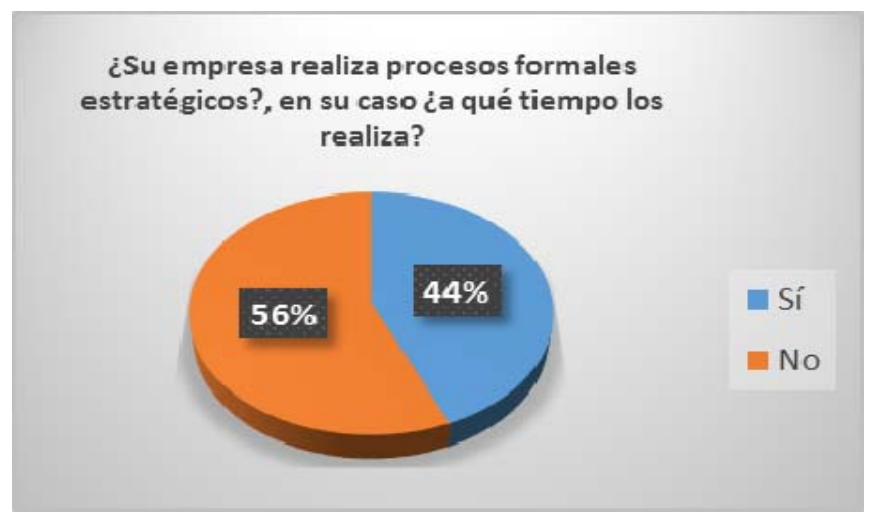

Fuente: los autores.

Los resultados arrojados en este caso hacen suponer un decrecimiento o la tendencia a mantenerse estáticas en sus actividades socioeconómicas, lo que puede explicar diferencia de competitividad con relación a las Mipymes que están bajo el sistema de asociatividad. Seguidamente se muestra el resultado de la misma pregunta en las Mipymes que cumplen con esta condición.

Tabla 3 Procesos formales estratégicos (asociadas)

\begin{tabular}{lcc}
\hline & Frecuencia & Porcentaje \\
\hline Sí & 46 & $56.7 \%$ \\
No & 20 & $56 \%$ \\
\hline NS NR & 3 & $4.3 \%$ \\
\hline TOTAL & 69 & $100,0 \%$ \\
\hline
\end{tabular}

Fuente: los autores. 


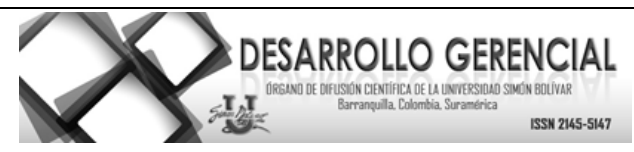

En la tabla 3, se observa que amparadas por un sistema de asociatividad, el $66.7 \%$ de las mypimes realiza procesos de planeación estratégica, el $29 \%$ no la realiza y un $4.3 \%$ no sabe. En la gráfica 3 se aprecia la distribución en porcentajes en función de los procesos formales estratégicos.

\section{Gráfica 3. Planeación estratégica formal.}

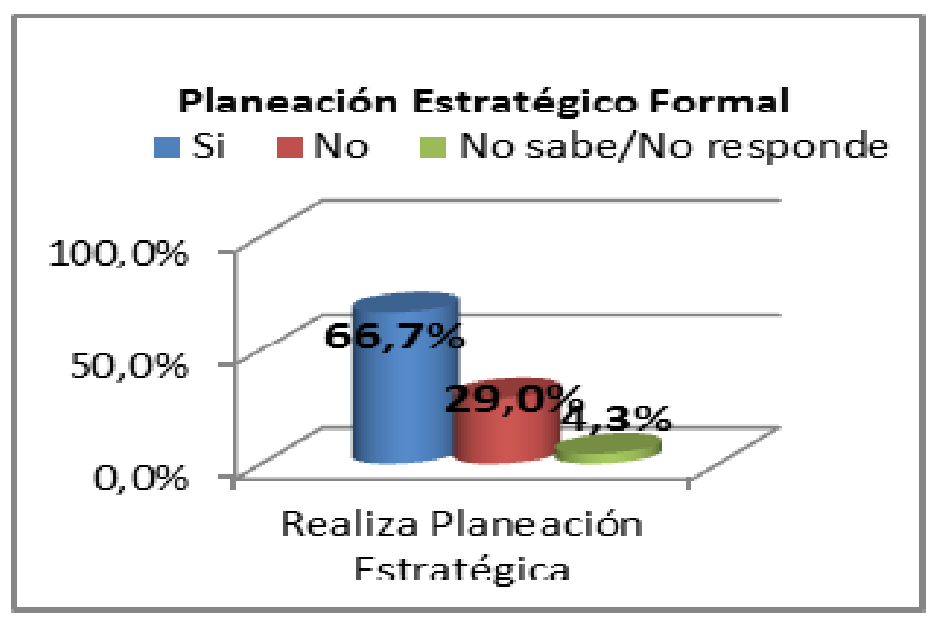

Fuente: Los autores.

Estos resultados indican una mejor aplicación de los procesos de planeación estratégica en las empresas con niveles de asociatividad que las que carecen de esta condición. Esto podría indicar un mejor de desempeño en sus tendencias de ventas a diferencia de las que no están en una cultura de interconexiones estratégicas.

Las gráficas que siguen podrían explicar un comportamiento diferenciador entre los dos casos.

Tabla 4 Tendencia de ventas años 2012 y 2013 (no asociadas)

\begin{tabular}{|l|c|}
\hline \multicolumn{1}{|c|}{ VARIABLE } & Frecuencia \\
\hline $\mathbf{2 0 1 2}$ & \\
\hline Aumentó & 50 \\
\hline Igual & 30 \\
\hline Disminución grave & 5 \\
\hline TOTAL & $\mathbf{8 5}$ \\
\hline $\mathbf{2 0 1 3}$ & \\
\hline Aumentó & 55 \\
\hline Igual & 27 \\
\hline Disminución grave & 3 \\
\hline TOTAL & $\mathbf{8 5}$ \\
\hline
\end{tabular}

Fuente: los autores. 


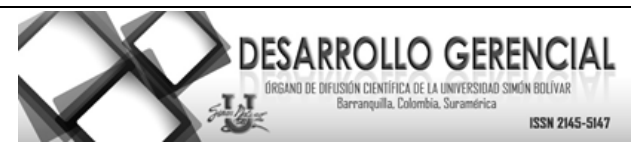

La tabla 4 evidencia la tendencia de ventas que muestran los resultados, y que aportarán una mejor comprensión para resolver la incógnita que es objeto de estudio, con relación a los datos de las Mipymes no asociadas.

Los resultados determinan que el 58.8\% (50 empresas) de los entrevistados admite un crecimiento en ventas; para el 35.3\% (30 empresas) es igual o invariable este comportamiento; para un 5.8\% (5 empresas) ha disminuido el movimiento de las ventas y para el resto no sabe/no responde. Mientras tanto el 55\% reporta para el 2013 un crecimiento; para el 27\% siguió igual; para el 3\% hubo disminución y el resto no sabe/no responde. En la gráfica 4 se puede observar la distribución porcentual.

Gráfica 4. Tendencia de ventas Mipymes no asociadas.

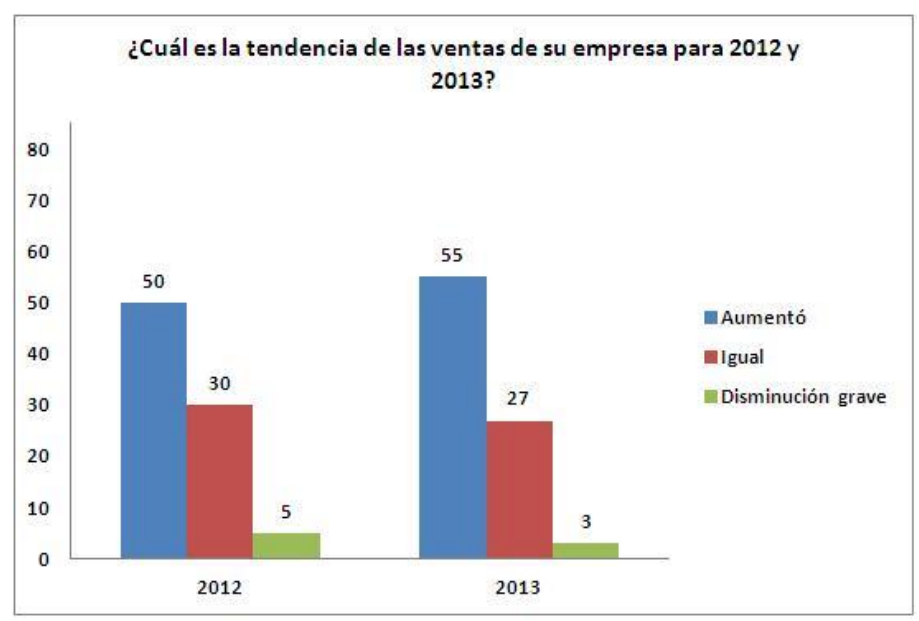

Fuente: los autores.

La anterior información refleja una leve mejoría de opinión en el 2013, en comparación al periodo anterior. Seguidamente se mostrarán los datos arrojados de la misma pregunta a los empresarios cuyas mipymes están bajo el sistema de asociatividad.

Tabla 5. Tendencia de ventas años 2012 y 2013 (asociadas)

\begin{tabular}{|c|c|}
\hline VARIABLE & Frecuencia \\
\hline 2012 & 41 \\
\hline Aumentó & 18 \\
\hline Igual & 6 \\
\hline Disminución grave & 4 \\
\hline NS NR & $\mathbf{6 9}$ \\
\hline TOTAL & \\
\hline 2013 & 47 \\
\hline Aumentó & 11 \\
\hline Igual & \\
\hline
\end{tabular}




\begin{tabular}{|c|c|}
\hline Disminución grave & 2 \\
\hline NS NR & 9 \\
\hline TOTAL & $\mathbf{6 9}$ \\
\hline
\end{tabular}

Fuente: los autores.

Los resultados determinan que el $59.4 \%$ de los entrevistados admite un crecimiento en materia de ventas; para el $26.1 \%$ es igual; para un $3.0 \%$ ha disminuido y el resto no sabe/no responde. Mientras que para el $68.1 \%$ reporta que ha habido crecimiento; para el $15.9 \%$ siguió igual; para el $3 \%$ hubo disminución y el resto no sabe/no responde. En la gráfica 5 se aprecia la tendencia marcada en los años 2012 y 2013.

\section{Gráfica 5. Tendencia en ventas de Mipymes asociadas}

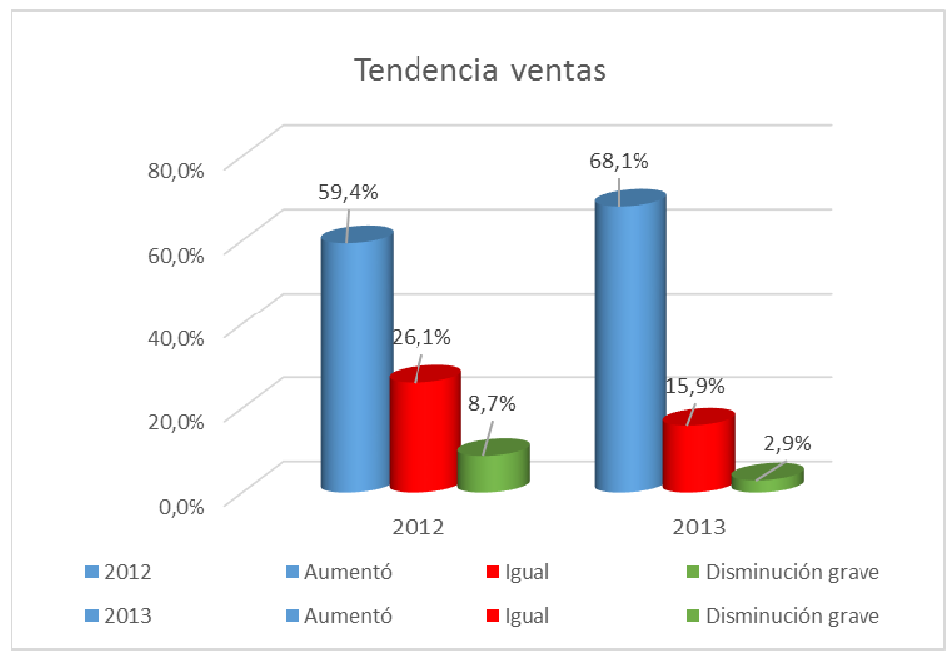

Fuente: los autores

Se puede confirmar con la información de los entrevistados que de las mipymes analizadas en este estudio las que presentan un mejor desempeño han sido las que están en modelo de asociatividad tal como lo explican los datos obtenidos del estudio.

\section{Prueba de comparación de dos proporciones}

Para comparar los dos grupos se empleó la prueba de comparación de dos proporciones, en función de las variables cualitativas: planea estrategias y tendencia de ventas. En la siguiente tabla de contingencia se muestra cuál es la proporción en cada uno de los grupos; es decir, las mipymes que están asociadas y aquellas que no lo están, con relación al hecho de planear o no. 


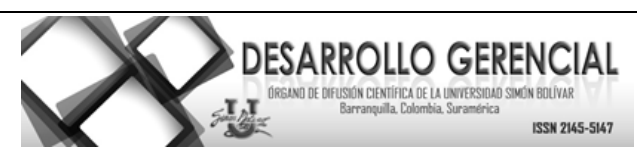

Tabla 6. Procesos formales estratégicos-tipo empresa

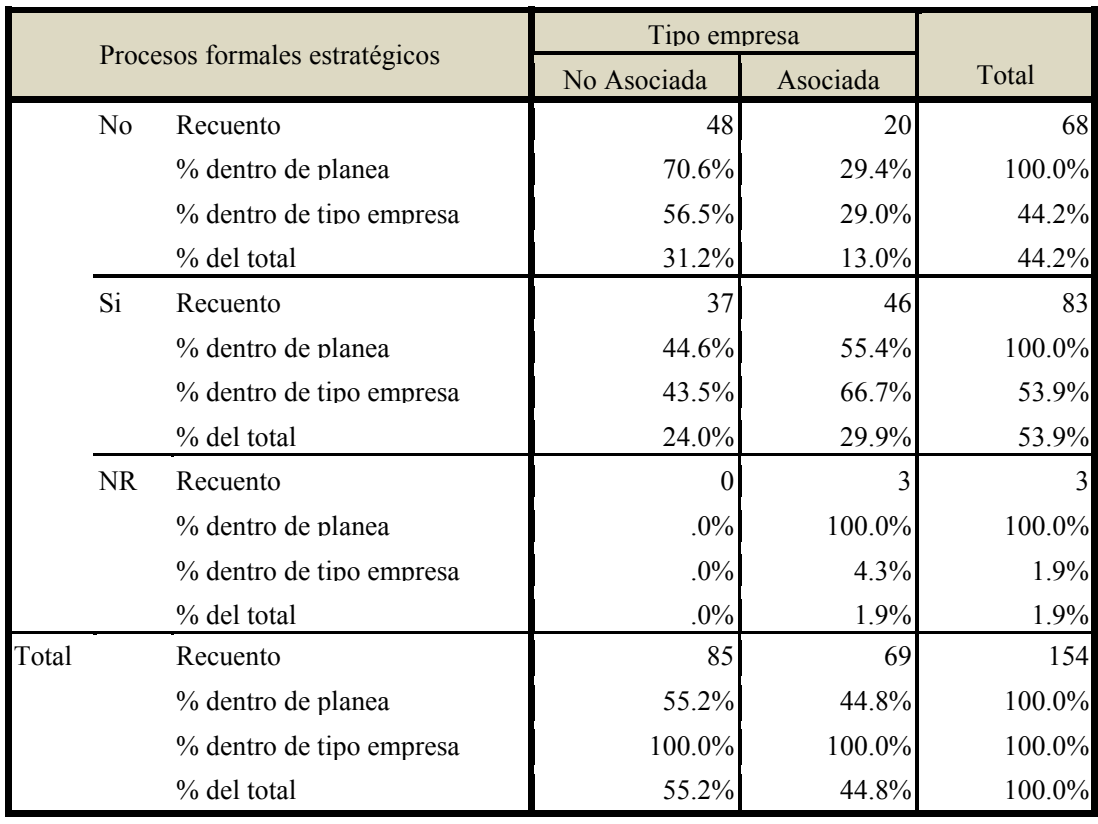

Fuente: Los autores

En la tabla 6 se puede apreciar que 46 empresas asociadas realizan procesos formales estratégicos, y 37 de las no asociadas también lo hacen. Así mismo, 20 empresas asociadas no desarrollan procesos formales estratégicos y 48 de las no asociadas tampoco lo hacen. En apariencia se aprecia que la mayoría de las empresas asociadas planean y que las no asociadas no planean. Ello haría sospechar una relación hacia el hecho de asociarse y desarrollar procesos formales estratégicos al menos a nivel descriptivo. Pero si en realidad queremos comprobar una relación, entonces debemos usar una Chi-cuadrado de Pearson, como a continuación se relaciona en la tabla 7 .

Tabla 7. Pruebas de Chi-cuadrado

\begin{tabular}{|l|c|c|c|}
\hline & Valor & $\mathrm{gl}$ & $\begin{array}{c}\text { Sig. asintótica } \\
\text { (bilateral) }\end{array}$ \\
\hline Chi-cuadrado de Pearson & $13.994^{\mathrm{a}}$ & 2 & .001 \\
Razón de verosimilitudes & 15.351 & 2 & .000 \\
Asociación lineal por lineal & 13.554 & 1 & .000 \\
N de casos válidos & 154 & & \\
\hline
\end{tabular}

a. 2 casillas $(33,3 \%)$ tienen una frecuencia esperada inferior a 5 . La frecuencia mínima esperada es 1,34 .

Si se atienden que la hipótesis nula es que no hay relación entre el hecho de estar asociados y desarrollar procesos formales estratégicos; si tenemos que la distribución teórica de Pearson está con 2 gl. Lo que indica que la probabilidad que la hipótesis nula sea cierta que tiende a cero (0); es decir, está por 


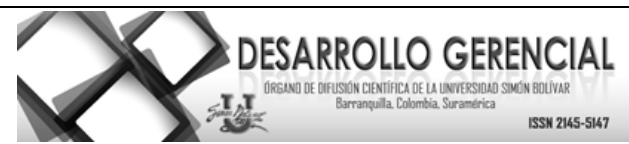

debajo de 0,05 (5\%); siendo tan poco probable la hipótesis nula, se rechazamos. Ello sugiere entonces que existe una relación entre el hecho de estar asociados y desarrollar procesos formales estratégicos.

Tabla 8. Tendencia de ventas años 2012-tipo de empresa

\begin{tabular}{|c|c|c|c|c|c|}
\hline \multirow{2}{*}{\multicolumn{3}{|c|}{ Tendencia de ventas }} & \multicolumn{2}{|c|}{ Tipo empresa } & \multirow{3}{*}{$\frac{\text { Total }}{91}$} \\
\hline & & & \multirow{2}{*}{$\frac{\text { No asociada }}{50}$} & \multirow{2}{*}{$\frac{\text { Asociada }}{41}$} & \\
\hline & Incrementó & Recuento & & & \\
\hline & & $\%$ dentro de vta. 2012 & $54.9 \%$ & $45.1 \%$ & $100.0 \%$ \\
\hline & & $\%$ dentro de tipo empresa & $58.8 \%$ & $59.4 \%$ & $59.1 \%$ \\
\hline & & $\%$ del total & $32.5 \%$ & $26.6 \%$ & $59.1 \%$ \\
\hline & Igual & Recuento & 30 & 18 & 48 \\
\hline & & $\%$ dentro de vta. 2012 & $62.5 \%$ & $37.5 \%$ & $100.0 \%$ \\
\hline & & $\%$ dentro de tipo empresa & $35.3 \%$ & $26.1 \%$ & $31.2 \%$ \\
\hline & & $\%$ del total & $19.5 \%$ & $11.7 \%$ & $31.2 \%$ \\
\hline & Disminución & Recuento & 5 & 6 & 11 \\
\hline & & $\%$ dentro de vta. 2012 & $45.5 \%$ & $54.5 \%$ & $100.0 \%$ \\
\hline & & $\%$ dentro de tipo empresa & $5.9 \%$ & $8.7 \%$ & $7.1 \%$ \\
\hline & & $\%$ del total & $3.2 \%$ & $3.9 \%$ & $7.1 \%$ \\
\hline & NR & Recuento & 0 & 4 & 4 \\
\hline & & $\%$ dentro de vta. 2012 & $.0 \%$ & $100.0 \%$ & $100.0 \%$ \\
\hline & & $\%$ dentro de tipo empresa & $.0 \%$ & $5.8 \%$ & $2.6 \%$ \\
\hline & & $\%$ del total & $.0 \%$ & $2.6 \%$ & $2.6 \%$ \\
\hline Total & & Recuento & 85 & 69 & 154 \\
\hline & & $\%$ dentro de vta. 2012 & $55.2 \%$ & $44.8 \%$ & $100.0 \%$ \\
\hline & & $\begin{array}{l}\% \text { dentro de tipo de } \\
\text { empresa }\end{array}$ & $100.0 \%$ & $100.0 \%$ & $100.0 \%$ \\
\hline & & $\%$ del total & $55.2 \%$ & $44.8 \%$ & $100.0 \%$ \\
\hline
\end{tabular}

Fuente: Los autores

En la tabla 8 se observa que con relación a la tendencia de ventas para el año 2012, 50 empresas no asociadas manifiestan haber tenido un incremento en los despachos, y 41 de las empresas asociadas expresan lo mismo. De igual forma podría sugerirse que si se acepta que un incremento en las ventas expresa la competitividad de las empresas, a simple vista se refleja que no hay relación entre el hecho de estar asociadas y la competitividad.

En la tabla 8 si se observa la significación asintótica, esta se encuentra por encima del 0,05 (5\%). En este sentido se puede señalar que no existe relación entre la competitividad de las empresas y el hecho de asociarse. Pero, por otro lado, en la tabla 9, como se verá más adelante, se muestra lo contrario. 
Tabla 9 Prueba de Chi-cuadrado

\begin{tabular}{|l|r|r|r|}
\hline & Valor & $\mathrm{gl}$ & \multicolumn{2}{|c|}{$\begin{array}{c}\text { Sig. asintótica } \\
\text { (bilateral) }\end{array}$} \\
\hline Chi-cuadrado de Pearson & $6.388^{\mathrm{a}}$ & 3 & .094 \\
Razón de verosimilitudes & 7.894 & 3 & .048 \\
Asociación lineal por lineal & 1.318 & & 1 \\
$\mathrm{~N}$ de casos válidos & 154 & & .251 \\
\hline
\end{tabular}

a. 3 casillas $(37,5 \%)$ tienen una frecuencia esperada inferior a 5. La frecuencia mínima esperada es 1,79 .

Si se atiende que la hipótesis nula es que no hay relación entre el hecho de estar asociados y tendencia a las ventas, y si tenemos que la distribución teórica de Pearson está con 3 gl., indicando que la probabilidad que la hipótesis nula sea cierta está por encima de 0,05 (5\%), siendo probable la hipótesis nula, se acepta; ello sugiere entonces que no existe una relación entre el hecho de estar asociados y la tendencia de ventas para el 2012.

En este sentido, si se observan los cambios producidos en la tendencia de ventas para el 2013, aun cuando sean mínimamente observables, de manera estadística son comprobables en la tabla 10; por ello se visualiza que este panorama cambia de manera significativa.

Tabla 10. Tendencia de ventas años 2013-tipo de empresa

\begin{tabular}{|c|c|c|c|c|}
\hline \multirow{2}{*}{\multicolumn{2}{|c|}{ Tendencia de ventas 2013}} & \multicolumn{2}{|c|}{ Tipo empresa } & \multirow[b]{2}{*}{ Total } \\
\hline & & No asociada & Asociada & \\
\hline \multirow[t]{4}{*}{ Incrementó } & Recuento & 55 & 47 & 102 \\
\hline & $\%$ dentro de vta. 2013 & $53.9 \%$ & $46.1 \%$ & $100.0 \%$ \\
\hline & $\%$ dentro de tipo empresa & $64.7 \%$ & $68.1 \%$ & $66.2 \%$ \\
\hline & $\%$ del total & $35.7 \%$ & $30.5 \%$ & $66.2 \%$ \\
\hline \multirow[t]{4}{*}{ Igual } & Recuento & 27 & 11 & 38 \\
\hline & $\%$ dentro de vta. 2013 & $71.1 \%$ & $28.9 \%$ & $100.0 \%$ \\
\hline & $\%$ dentro de tipo empresa & $31.8 \%$ & $15.9 \%$ & $24.7 \%$ \\
\hline & $\%$ del total & $17.5 \%$ & $7.1 \%$ & $24.7 \%$ \\
\hline \multirow{4}{*}{$\begin{array}{l}\text { Disminución } \\
\text { grave }\end{array}$} & Recuento & 3 & 2 & 5 \\
\hline & $\%$ dentro de vta. 2013 & $60.0 \%$ & $40.0 \%$ & $100.0 \%$ \\
\hline & $\%$ dentro de tipo de empresa & $3.5 \%$ & $2.9 \%$ & $3.2 \%$ \\
\hline & $\%$ del total & $1.9 \%$ & $1.3 \%$ & $3.2 \%$ \\
\hline \multirow[t]{4}{*}{ NR } & Recuento & 0 & 9 & 9 \\
\hline & $\%$ dentro de vta. 2013 & $.0 \%$ & $100.0 \%$ & $100.0 \%$ \\
\hline & $\%$ dentro de tipo empresa & $.0 \%$ & $13.0 \%$ & $5.8 \%$ \\
\hline & $\%$ del total & $.0 \%$ & $5.8 \%$ & $5.8 \%$ \\
\hline
\end{tabular}




\begin{tabular}{|c|c|c|c|c|}
\hline \multirow[t]{4}{*}{ Total } & Recuento & 85 & 69 & 154 \\
\hline & $\%$ dentro de vta. 2013 & $55.2 \%$ & $44.8 \%$ & $100.0 \%$ \\
\hline & $\%$ dentro de tipo empresa & $100.0 \%$ & $100.0 \%$ & $100.0 \%$ \\
\hline & $\%$ del total & $55.2 \%$ & $44.8 \%$ & $100.0 \%$ \\
\hline
\end{tabular}

Fuente: Los autores

En la tabla 10 se observa que hay un ligero incremento en el número de empresas asociadas para el año 2013 en relación con el 2012, al menos en apariencia. Lo mismo ocurre con las no asociadas; pero en la disminución grave de 6 empresas asociadas, que manifestaron en el 2012 estar en esa situación, bajó a 3 empresas. Mientras el número de empresas asociadas que manifestaron estar igual, bajó de 18 a 11. Con lo anterior, en la tabla 10 se refleja esta diferencia de manera significativa estadísticamente.

Tabla 11. Pruebas de Chi-cuadrado

\begin{tabular}{|l|c|c|c|}
\hline & Valor & $\mathrm{gl}$ & $\begin{array}{c}\text { Sig. asintótica } \\
\text { (bilateral) }\end{array}$ \\
\hline Chi-cuadrado de Pearson & $15.065^{\mathrm{a}}$ & 3 & .002 \\
Razón de verosimilitudes & 18.592 & 3 & .000 \\
Asociación lineal por lineal & 2.764 & 1 & .096 \\
N de casos válidos & 154 & & \\
\hline
\end{tabular}

a. 4 casillas $(50,0 \%)$ tienen una frecuencia esperada inferior a 5 . La frecuencia mínima esperada es 2,24 .

Si bien la hipótesis nula expresa que no hay relación entre el hecho de estar asociados y tendencia a las ventas, desde la distribución teórica de Pearson está con 3 gl., y señalando que la probabilidad que la hipótesis nula sea cierta está por debajo de 0,05 (5\%), siendo poco probable la hipótesis nula, la rechazamos; ello sugiere entonces que existe una relación entre el hecho de estar asociados y la tendencia de ventas para el 2012.

Podría suponerse que un hecho que influye en la posibilidad de rechazar una hipótesis, para luego aceptarla está relacionado con el tiempo que llevan las empresas asociadas, al menos es un año donde se tiene la posibilidad de fortalecerse.

\section{4.- DISCUSIÓN}

El estudio muestra que el sistema de asociatividad en las mipymes ha influido favorablemente en su desempeño de gestión estratégica y en las tendencias de crecimiento en ventas de un periodo a otro, a diferencia de las que no han contado con este modelo. Ello se ve reflejado con la aplicación del estadígrafo de Chi-cuadrado de Pearson, en donde las variables de desarrollo de procesos formales 


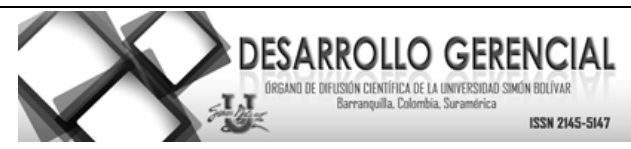

estratégicos y el hecho de estar asociadas, presentan una correlación, en tanto que está por debajo del 5\% $(\mathrm{p}<0,05)$.

El hecho de asociarse o no asociarse no implica que las mipymes que sí lo están van a tener ventaja competitiva, en comparación con aquellas que no están asociadas, en tanto que como se aprecia en las tablas 4 y 5, el aumento en ventas comparadas para los años 2012 y 2013 en cada uno de los grupos aumentó de manera similar; pero de igual forma y con evidencia estadística, a pesar de presentar ese fenómeno de incremento directo, estadísticamente se evidencia que para el año siguiente las diferencias se pueden apreciar.

El comportamiento de las Mipymes reflejado en las tablas y gráficas de los resultados, hace suponer que una Mipymes en un sistema de asociatividad recibe un número de beneficios relacionados con el fortalecimiento de sus recursos y capacidades debido a las sinergias que se realizan entre asociados y profesionales que conforman el gobierno interorganizacional en los modelos de asociatividad.

Cabe resaltar que aunque el porcentaje de Mipymes no asociadas que utilizan procesos de planeación estratégica es inferior al de las asociadas, ello sugiere que esta herramienta, como estrategia competitiva, es de vital importancia para el crecimiento empresarial, siendo coherentes con que los rendimientos obtenidos por las empresas son dependientes de la orientación estratégica que se siga (Spanos et al., 2004; Aragón y Sánchez, 2005; Rubio y Aragón, 2009).

De igual forma puede confirmarse que una de las formas de conocer el crecimiento se debe reflejar en las ventas por periodos; luego partiendo de las opiniones de los empresarios prevalece el crecimiento en gran porcentaje entre las mipymes analizadas mientras que en decrecimiento o con tendencia a permanecer estables son minoría; pero predominan con mayor éxito las empresas bajo parámetros de asociatividad como se ha podido demostrar en los estudios. Por otro lado, se puede contrastar con lo que confirman Aragón y Rubio (2005): la cooperación representa una alternativa estratégica que permite alcanzar el tamaño competitivo en ciertas actividades sin necesidad de fusionarse y manteniendo la independencia jurídica y patrimonial.

Además, un estudio podría aportar insumos con los que en un futuro se pudiera ampliar la motivación de sus propietarios de modo que puedan participar en sistemas de asociatividad y así mejorar su desarrollo empresarial.

De los resultados se deduce que las Mipymes en asociatividad pueden obtener beneficios en movilidad de recursos valiosos de manera relevante, como agremiación; en cierto modo, esta situación es consistente con lo expuesto en Aragón y Rubio (2005) en el sentido de que puedan alcanzar el tamaño competitivo. 


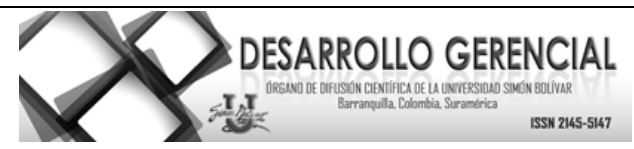

\section{5.- REFERENCIAS}

Aragón, A. y Rubio, A. (2005, mayo-agosto). Factores explicativos del éxito competitivo: el caso de las Pymes del estado de Veracruz. Contaduría y Administración, 216, 35-69.

Aragón, A. y Sánchez, G. (2005). Strategic orientation, management characteristics, and performance: A study of Spanish SMEs. Journal of Small Business Management, 43(3), 287.

Armijo, M. (2009). Manual de Planificación Estratégica e indicadores de desempeño en el sector público. Área de Políticas Presupuestarias y Gestión Pública. Santiago de Chile: Ilpes/Cepal.

Deeds, D.L. y Hill, C.W. (1996). Strategic alliances and the rate of new product development: an empirical study of entrepreneurial biotechnology firms. Journal of Business Venturing, 11(1), 4155.

Gálvez, E., Cuéllar, K., Restrepo, C., Bernal, C. y Cortés, J. (2012). Análisis estratégico para el desarrollo de la Mipyme en Iberoamérica. Cali, Colombia: Universidad del Valle.

González, J. y Rosero, M. (2011). Análisis de estructura, conducta y desempeño del subsector de la confitería en el área metropolitana de Cali 2002-2010. Revista Entramado, 7 (2), 72-84.

Grant, R. M. (1996). Dirección estratégica: conceptos, técnicas y aplicaciones. Madrid, España: Civitas.

Gnjidić, V. (2014). Researching the dynamics of Miles and Snow's strategic typology. Management: Journal of Contemporary Management Issues, 19 (1), 93-117.

Hernández, R., Fernández, C. y Baptista, M. (2014). Metodología de la investigación (6. ${ }^{a}$ Ed.). México: McGraw-Hill.

McDaniel, S.W. y Kolari, J.W. (1987). Marketing Strategy implications of the Mile and Snow Strategic Typology. Journal of Marketing, 5 (4), 51-70.

Ministerio de Comercio, Industria y Turismo (s.f.). Reporte de Mipymes No. 3. Recuperado de file://C:/Users/emprendimiento/Downloads/ReporteMipymes03\%20(1).pdf.

Montes, A. y Sabater, R. (2002). Alianzas estratégicas: modelo para la gestión del proceso de negociación. Revista Empresa, 84-99.

Ojeda, J. (2009). La cooperación empresarial como estrategia de las Pymes del sector ambiental. Estudios Gerenciales, 25 (110), 39-61.

Pleshko, L.P. (2006, January). Product growth strategies of the miles and snow strategic groups. In Allied Academies International Conference. Academy of Strategic Management. Proceedings, 5 (1), 15.

Desarrollo Gerencial, 7 (1) pp. 102 - 121 Enero-Junio 2015. ISSN: 2145-5147 (On Line). Universidad Simón Bolívar. 


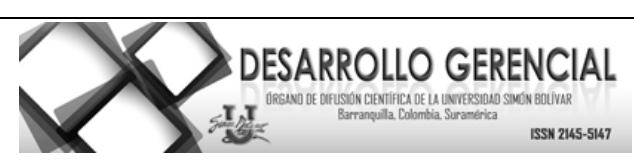

Porter, M.E. (1980). Competitive strategy: Techniques for analyzing industries and competition. New York, 300 .

Rubio, A. y Aragón, A. (2009). SMEs competitive behavior: strategic resources and strategies. Management Research: Journal of the Iberoamerican Academy of Management, 7 (3), 171-190.

Saavedra, G. y Hernández, Y. (2008). Caracterización e importancia de las Mipymes en Latinoamérica: Un estudio comparativo. Actualidad contable faces, 11(17), 122-134.

Slater, S. y Narver, J. (1993). Product-market strategy and performance: an analysis of the Miles and Snow strategy types. European journal of marketing, 27(10), 33-51.

Spanos, Y.E., Zaralis, G. y Lioukas, S. (2004). Strategy and industry effects on profitability: Evidence from Greece. Estrategia Management Journal, 25, 139-165.

Varela R. y Liyis F. (2013). Dinámica empresarial colombiana. Bogotá (Colombia): Global Entrepreneurship Monitor (GEM).

Wright, H., Wedaman, W., Morand y Scholey. (1991). Subcellular localization and sequence of seaurchin kinesin heavy chain: evidence for its association with membranesin the mitotic apparatus and interphase cytoplasm. Cell Biol, 113, 817-833. 